\title{
Further validation of the questionnaire for eudaimonic well-being (QEWB)
}

\author{
Lusilda Schutte*, Marié P Wissing and Itumeleng P Khumalo
}

\author{
* Correspondence: \\ 13012584@nwu.ac.za \\ Department of Psychology, \\ North-West University, \\ Potchefstroom, South Africa
}

\begin{abstract}
Objective: The aim of this study was to explore the structural and external validity of the recently developed Questionnaire for Eudaimonic Well-Being (QEWB), scrutinising the parcelling approach used by Waterman and colleagues to confirm the unidimensional structure of the scale.

Method: A multicultural South African student sample $(n=325)$ was used. Parceland item-level confirmatory factor analysis and item-level exploratory factor analysis were conducted to explore the scale's structural validity. External validity was examined via attenuation corrected correlations with scores on criterion measures.

Results: On parcel-level a one-dimensional structure fitted well, but since the assumption of unidimensional parcels was violated, the use of parcelling was contraindicated. Item-level analyses revealed a multidimensional factor structure. The scale showed good convergent and discriminant validity.

Conclusions: The QEWB shows potential for future use, given that the multidimensionality of the scale is acknowledged. Some items may need revision.

Keywords: Questionnaire for eudaimonic well-being; Eudaimonia; Validation; Item parcelling; Factor structure; Measurement
\end{abstract}

\section{Background}

Discourse about what constitutes psychosocial well-being is receiving extensive attention in current literature (e.g. Kashdan et al. 2008). Two main streams of thought are often distinguished, namely the hedonic view, which focuses on "feeling good" (Diener 1984; Kahneman et al. 1999) and the eudaimonic perspective, which is concerned with "functioning well" (Deci and Ryan 2008; Huta and Ryan 2010; Waterman 1993; Waterman et al. 2010). In the past, well-being was particularly studied from a hedonic perspective with little attention to the eudaimonic view. Recently, however, research on eudaimonic well-being (EWB) is gaining momentum, and the need for assessment measures for the evaluation of facets of eudaimonic well-being is noted (Waterman et al. 2010).

The endeavour to study EWB depends on the development of high quality assessment measures for the evaluation of facets of EWB (Waterman et al. 2010). The Questionnaire for Eudaimonic Well-Being (QEWB) was developed by Waterman et al. with this purpose in mind. The QEWB was developed as an operational definition of EWB and items that Waterman et al. (2010) considered to be closely related to contemporary philosophical

C 2013 Schutte et al.; licensee Springer. This is an Open Access article distributed under the terms of the Creative Commons Attribution License (http://creativecommons.org/licenses/by/2.0), which permits unrestricted use, distribution, and reproduction in any medium, provided the original work is properly cited. 
views on eudaimonia were included. The questionnaire included items from both the objective understanding of eudaimonia (i.e., qualities associated with eudaimonic functioning, such as the pursuit of excellence and self-realisation) and from the subjective stance (i.e., subjective experiences of eudaimonia, such as feelings that one is engaged in activities that are personally expressive). The interrelated categories of self-discovery, perceived development of one's best potentials, sense of purpose and meaning in life, investment of significant effort in pursuit of excellence, intense involvement in activities, and enjoyment of activities as personally expressive formed the basis for item formulation and content.

\section{Validation of the QEWB}

Waterman et al. (2010) followed Simms' (2008) guidelines for proper scale construction in the development of the QEWB. Simms proposed that scale development involves three stages: firstly, the substantive validity phase, where theory-informed conceptualisations of the construct are formed and the need for the scale is established; secondly, the structural validity phase, where the psychometric properties of items are evaluated and provisional scales are created; and thirdly, the external validity phase, where convergent, discriminant, and criterion-related validity is assessed. In their validation of the QEWB, Waterman et al. (2010) gave extensive consideration to Simms' first and third phases. Regarding the first, they provided a substantial discussion of the theoretical definition of EWB that was to be operationalised by the QEWB, and concerning the latter, they tested a number of hypotheses about relationships between scores on the QEWB and scores on indicators of the development of identity commitments, other forms of well-being, identity exploration, personality traits, and positive and negative psychological functioning. However, the structural validity was attended to only briefly.

In their brief discussion of the structural validity of the QEWB, Waterman et al. (2010) provided descriptive statistics for the scale's total scores, but not for individual items. The item-level psychometric examination of the scale using descriptive statistics is valuable as it can, for example, provide information regarding univariate normality (Bandalos and Finney 2010). Cronbach's alpha coefficient was provided to indicate the internal consistency reliability of the scale, but additional measures, such as the mean interitem correlation and item-total correlations, will add further information.

Regarding the QEWB's factor structure, Waterman et al. (2010) conducted analyses to confirm the scale's unifactorial structure, without providing any motivation for the hypothesised unidimensionality. In fact, they thoroughly discussed the six interlinked aspects of EWB that formed the basis of item formulation. We considered that the specified aspects may be interlinked, but still distinct. Therefore we hypothesised that a multidimensional factor structure with intercorrelated factors may be a possibility.

Furthermore, we have questions about the analytical approach Waterman et al. (2010) used to confirm the scale's unidimensionality. They applied confirmatory factor analysis (CFA) to verify their hypothesis that the QEWB has a unidimensional structure, but no exploratory factor analysis (EFA) was reported to investigate possible multidimensionality. In the CFA, which was applied via structural equation modelling, 
Kline's (2005) recommendation that "no more than 5-6 indicators should be used to define a latent variable" (Waterman et al. 2010, p. 52) was followed. Therefore, Waterman et al. used parcelled indicators where adjacent items were summed to form five parcels. However, parcelling in this instance is controversial because parcelling "assumes that items within each parcel are unidimensional” (Kline 2011, p. 181). Kline (2011) indicated that:

Parceling is not recommended if unidimensionality cannot be assumed. Specifically, parceling should not be part of an analysis aimed at determining whether a set of items is unidimensional. This is because it is possible that parceling can mask a multidimensional factor structure in such a way that a seriously misspecified model may nevertheless fit the data reasonably well. (pp. 181-182)

Waterman et al. parcelled adjacent items, but gave no theoretical justification for or statistical test of the unidimensionality of items that were combined into parcels. They stated that item formulation was based on six interrelated categories of EWB, and on face value it seemed to us as if items that were combined into parcels often belonged to different theoretical categories. From a theoretical point of view, this made us question the viability of the assumption of unidimensionality within parcels. A first step would be to empirically examine the feasibility of the assumption of unidimensionality of the parcels. If the assumption proves to be viable, the parcelling approach suffices for the sample under study. If not, additional approaches, such as item-level EFAs, should be used to explore the scale's dimensionality.

In addition to finding the feasibility of parcelling questionable, the necessity of using parcelling to conduct CFAs deserves scrutinisation. The rationale for parcelling lies in the fact that ordinal data (such as Likert scale data) often does not satisfy the assumptions of continuity and multivariate normality that various estimation techniques in structural equation modelling, such as maximum likelihood estimation, rely on, while total scores of ordinal items tend to be continuous and normally distributed (Kline 2011). However, Blunch (2008) stated that ordinal variables can be treated as if they were normally distributed interval scaled variables if they can take on at least five possible values, if their skewness and kurtosis values are close to zero, and if a possible limited skewness goes to the same side for all variables. These pointers need to be evaluated and if they are satisfied, commonly used estimation methods in CFA, like maximum likelihood, can be applied and parcelling would not be needed.

Waterman et al. (2010) correlated scores on the QEWB with scores on a variety of other scales to establish the external validity of the scale, which is Simms' (2008) third stage of scale development. These scales covered a broad range of psychological functioning, but only two came from the well-being research: one tapping so-called "subjective well-being" (which concerns positive emotions and life satisfaction) and the other "psychological well-being" (which involves functioning well). It will be useful to correlate scores on the QEWB with scores on a wider range of well-being scales to further explore the nomological net of EWB as operationalised by the QEWB in order to ascertain the scale's convergent and discriminant validity.

The validation study of Waterman et al. (2010) was done using students from the USA. Positive psychologists are often critiqued for focusing mainly on developed, Western 
countries in their research (Selin and Davey 2012). This study contributes by examining the validity of the QEWB for a sample from South Africa, a developing African country.

\section{The present study}

The first aim of this research was to explore the structural validity of the QEWB. This involved an investigation of the psychometric properties of the individual items, the scale's internal consistency reliability, the unidimensional factor structure proposed by Waterman et al. (2010), as well as possible alternative factor structures. In particular, our goal was to scrutinise the parcelling approach used by Waterman et al. to confirm the unidimensional structure of the scale. Based on theoretical considerations and our discomfort with the psychometric procedures used by Waterman et al. to confirm the scale's unidimensionality, we hypothesised that the unidimensional factor structure that Waterman et al. confirmed through parcelling would not optimally explain the structure of the items. Although a single higher-order factor may underlie the QEWB, our conjecture was that item-level exploratory and confirmatory techniques would reveal a multidimensional factor structure. On the basis of the theoretically intended composition of EWB according to the model of Waterman et al., the following components underlying its theoretical framework are (or ought to be) operationalised in its meaure: self-discovery, perceived development of one's best potentials, sense of purpose and meaning in life, investment of significant effort in pursuit of excellence, intense involvement in activities, and enjoyment of activities as personally expressive.

Our second aim was to explore the scale's external validity (convergent and discriminant) with specific focus on correlations between scores on the QEWB and scores on other well-being scales as well as a measure of psychological dysfunction. Our hypotheses were that scores on the QEWB would have high positive correlations with scores on other measures in the family of EWB to confirm convergent validity and moderate positive correlations with scores on measures of hedonic well-being, a moderate negative correlation with scores on a depression scale, and a small negative correlation with scores on a measure of search for meaning (which was found to be slightly negatively correlated with meaning in life, an aspect of eudaimonic well-being [Steger et al. 2006]), to establish discriminant validity.

\section{Method}

\section{Research design and participants}

This was a quantitative study using a cross-sectional questionnaire survey with a sample of convenience. The sample $(n=325$, Male $=80$, Female $=242$, three unspecified $)$ consisted of students from a South African university. The average age in years was $21.03(S D=4.09)$, and it ranged between 18 and 54 years. South Africa's multicultural, multilingual demographical composition was reflected in the sample, where $18 \%$ of the sample indicated English as their native language, while the rest selected Setswana (18\%), Afrikaans (7\%), or Other (55\%) as their native language, or did not answer (1\%). The "Other" language category could one of the other eight of the eleven South African official languages, or any other language. The language of tuition is English at two of the three sites where data were collected, therefore it can be assumed that participants from those sites were sufficiently fluent in English. At the third site used for data 
collection, the language of tuition is both English and Afrikaans. At this site, participants had the choice to complete the survey in either English or Afrikaans. Only the data for group who completed the survey in English is included in this study, and we can assume that these participants were sufficiently fluent in English.

\section{Measures}

The QEWB was administered as part of a battery of psychological well-being and dysfunction scales that were used to determine the external validity the scale. For each of the criterion measures, Cronbach's alpha coefficient, for which values larger than .70 are often deemed adequate (Kline 2011), is reported as a measure of internal consistency. The comparative fit index (CFI), for which values larger than .95 are considered a sign of good fit, and the root mean square error of approximation (RMSEA) with its associated $90 \%$ confidence interval (CI), for which values below .08 show that the fit is acceptable, are reported as indicators of structural validity (Bandalos and Finney 2010).

\section{QEWB (Waterman et al. 2010)}

The QEWB consists of 21 Likert scale items and was developed to measure well-being in a manner consistent with how it is conceptualised in the model of Waterman et al. (2010), based within the eudaimonist philosophy. Seven of the items are phrased in a negative direction and need reversed scoring. The original QEWB by Waterman et al. requests participants to rate their agreement with each statement on a scale ranging from 0 (strongly disagree) to 4 (strongly agree). In this study, a scale ranging from 1 (strongly disagree) to 7 (strongly agree) was used in line with procedures in the Eudaimonic and Hedonic Happiness Investigation project (cf. Delle Fave et al. 2011) for which this study serves as a step in measurement validation for the next phase. Weijters et al. (2010) stated that the use of seven response options is acceptable when working with student samples, and as will be noted, most other measures in this study also used 7-point scales. Waterman et al. showed sufficient reliability $(\alpha=.86)$ and convergent, discriminant, construct, and incremental validity for the scale for an ethnically diverse American student sample. We could not find other validation studies of the QEWB.

\section{Mental Health Continuum - Short Form (Keyes 2002, 2006, 2009)}

This 14-item questionnaire measures positive mental health. Respondents rate the frequency of each statement occurring in the past month on a Likert scale ranging from 0 (never) to 5 (every day). It includes three items of emotional well-being in terms of positive affect and satisfaction with life, five items of social well-being based on Keyes' (1998) model of social well-being, and six items of psychological well-being as described in Ryff's (1989) model. Keyes et al. (2008) validated the Setswana version of this scale and found good psychometric properties. Lamers et al. (2011) confirmed the cross-cultural potential of the scale when they found good psychomteric properties of the MHC-SF among a representative Dutch sample. In the present study, Cronbach's alpha coefficients were .77 (Emotional Well-Being subscale), .72 (Social Well-Being subscale), and 79 (Psychological Well-Being subscale). Fit indices for CFA resulted in a CFI of .88 and an RMSEA of .084 (CI = $[.072, .096])$. 
Satisfaction with Life Scale (Diener et al. 1985)

This instrument is intended to measure a respondent's own assessment of the satisfaction with his or her life as a whole on a cognitive-judgemental level and consists of five 7-point Likert-type items, with anchor labels ranging from 1 (strongly disagree) to 7 (strongly agree). Diener et al. (1985) showed that the scale had good psychometric properties $(\alpha=.87)$ for samples of mainly American students, while construct validity and cross-cultural applicability of the scale were reported extensively in Pavot and Diener (1993, 2008). Within the South African context, Wissing and Van Eeden (2002) obtained sufficient reliability levels $(\alpha=.70-.86)$ and construct validity of the English version of the scale in a multicultural South African sample, while Wissing et al. (2010) showed acceptable reliability $(\alpha=.67)$ and construct validity for the Setswana version of the scale. The Cronbach's alpha coefficient was .74 in the present study. For the CFA the CFI was .98 and the RMSEA $.066(\mathrm{CI}=[.017, .115])$.

\section{Meaning in Life Questionnaire (Steger et al. 2006)}

This scale contains two five-item subscales measuring presence of and search for meaning in life, respectively. Respondents rate their degree of agreement on a 7-point Likert scale, ranging from 1 (absolutely untrue) to 7 (absolutely true). Steger et al. (2006) showed that the two subscales were reliable for mainly student samples, with the Cronbach's alpha values for the Presence subscale ranging from .82 to .86 and for the Search subscale from .86 to .87 . They also indicated construct, convergent, and discriminant validity of the instrument. The reliability and validity of translated versions of this scale in other countries have been showed, for example among Chinese (Liu and Gan 2010; Wang and Dai 2008) and Portuguese (Simões et al. 2010) groups. We could not find any applications of this scale within the South African context. In the present study, Cronbach's alpha coefficients were .85 (Presence subscale) and .85 (Search subscale). Fit indices for CFA resulted in a CFI of .95 and an RMSEA of $.076(\mathrm{CI}=[.058, .094])$.

\section{Sense of Coherence Scale (Antonovsky 1987, 1993)}

This 29-item scale measures an individual's cognitive self-evaluation of the world and his or her life in it, with regard to the extent that it is experienced as comprehensible, manageable, and meaningful, using a 7-point Likert scale with different semantic anchors for each item. Antonovsky (1993) indicated a high level of content, face, and criterion validity, with Cronbach's alpha scores in 26 studies ranging from .82 to 95 . Strümpfer and Wissing (1998) reported reliability indices ranging from .52 to .97 , with an average alpha of .87 in 27 South African studies implementing the English version. Wissing et al. (2010) showed that the Setswana version of the scale holds only moderate promise for applicability. Although the Cronbach's alpha was acceptable $(\alpha=.70)$ and the one-factor structure confirmed, interitem correlations were low and only $11.8 \%$ of the variance was explained by the single factor with particularly low loadings on items 5 and 20. They suggested that future research should be conducted in this regard. Despite problematic psychometric properties, this measure is included for purposes of this validation study, because there are very few translated measures from an EWB perspective available in South Africa, and because previous research on the instrument (Antonovsky 1993) attests to its applicability in various cultural contexts. The 
Cronbach's alpha coefficient was .82 in the present study. For the CFA the CFI was .54 and the RMSEA .084 $(\mathrm{CI}=[.079, .089])$.

Patient Health Questionnaire-9 (Kroenke et al. 2001)

This nine-item Likert scale measure consists of the actual nine DSM-IV criteria for diagnosis of a major depressive episode and can be used as a self-administered screening instrument to establish the diagnosis of depressive disorders and determine the severity of depressive symptoms. Respondents rate whether each symptom occurred 0 (not at all) to 3 (nearly every day) over the past two weeks. Kroenke et al. (2001) found the measure to have sufficient reliability ( $\alpha=.86-.89)$, specificity, sensitivity and validity in a sample of American primary care and obstetrics-gynaecology clinic patients. A vast number of studies have shown the validity of the scale in various populations, for example among Nigerian students (Adewuya et al. 2006) and Brazilian women (De Lima Osório et al. 2009). Botha (2011) showed that the English version of the scale was valid and reliable $(\alpha=.86)$ for a multicultural South African sample. The Cronbach's alpha coefficient was .82 in the present study. For the CFA the CFI was .93 and the RMSEA $.075(\mathrm{CI}=[.055, .095])$.

\section{Procedure}

The battery was compiled by combining the selected scales into a response booklet. Authorities of the sites where data was gathered were contacted to obtain permission, discuss ethical considerations, and make practical arrangements for data gathering. The lecturers and research assistants who helped as fieldworkers gathered the data, under the supervision of the researchers, in supervised class settings. Students from the selected sites volunteered to participate, and no monetary or academic credit remuneration was offered to them.

\section{Ethical considerations}

This project formed part of the FORT 3 project that had been approved by the Ethics Committee of the North-West University, South Africa, with project number NWU 00002-07-A2. Written informed consent was obtained from respondents prior to participation and responses were anonymous. Opportunities for debriefing were provided.

\section{Data analysis}

IBM SPSS Statistics 20 was used for all statistical analyses, except for the CFAs, for which IBM SPSS Amos 20 was used. Descriptive statistics (mean, standard deviation, skewness, kurtosis, interitem correlations, and item-total correlations) and reliability coefficients (Cronbach's alpha and average interitem correlation) were calculated. QEWB scores were compared for gender (independent t-tests) and age (ANOVA). CFAs were conducted via structural equation modelling (the confirmatory option) to establish construct validity, using both parcelled indicators, similar to Waterman et al. (2010), and the individual items as indicators. The proposed unidimensional structure of the scale was further tested with principal component analysis. EFAs were conducted to explore the multidimensionality of the scale. External validity (convergent and discriminant) was explored by examining the attenuation corrected correlational patterns between scores on the attained QEWB factors and scores on other measures in the 
family of EWB, measures more prominently associated with hedonic well-being, a measure of search for meaning, and a measure of depression. The attenuation correction was done by dividing the observed correlation coefficient by the square root of the product of the two scales' Cronbach's alpha values to compensate for the unreliability of the measures. However, caution should be applied when interpreting these corrected correlations, since alpha is a lower boundary for reliability, and division by alpha will then lead to overestimates of the corrected correlation (Bryant et al. 2007).

\section{Results}

Descriptive statistics and reliability analysis for the QEWB

As an initial exploration of the data, we calculated descriptive statistics of the individual items. After the calculation of reliability measures for the scale, descriptive statistics were also computed for the scale's total score.

\section{Item-level descriptive statistics}

Following Clark and Watson (1995), we started our investigation by inspecting the response distributions of the individual items before conducting more complex structural analyses. Blunch (2008) suggested that good items for summated scales require large variances, expected values near the middle value, positive intercorrelations of similar

Table 1 Item-Level Descriptive Statistics for Scores on the QEWB

\begin{tabular}{|c|c|c|c|c|c|c|c|}
\hline Item & Range & $M$ & $S D$ & Skew & Kur & ITC & $a$ if item is deleted \\
\hline 1 & $1-7$ & 4.80 & 1.63 & -0.54 & -0.23 & .41 & .79 \\
\hline 2 & $1-7$ & 5.02 & 1.75 & -0.79 & -0.13 & .49 & .79 \\
\hline 3 & $1-7$ & 3.99 & 2.03 & 0.04 & -1.17 & .13 & .81 \\
\hline 4 & $1-7$ & 5.72 & 1.37 & -1.09 & 0.77 & .52 & .79 \\
\hline 5 & $1-7$ & 6.15 & 1.29 & -1.85 & 3.36 & .42 & .79 \\
\hline 6 & $1-7$ & 5.60 & 1.53 & -1.39 & 1.64 & .58 & .78 \\
\hline 7 & $1-7$ & 5.53 & 1.80 & -1.13 & 0.16 & .33 & .80 \\
\hline 8 & $1-7$ & 6.06 & 1.19 & -1.52 & 2.48 & .37 & .80 \\
\hline 9 & $1-7$ & 4.85 & 1.81 & -0.68 & -0.45 & .52 & .79 \\
\hline 10 & $1-7$ & 4.64 & 2.10 & -0.47 & -1.11 & .03 & .82 \\
\hline 11 & $1-7$ & 5.24 & 1.93 & -0.84 & -0.55 & .42 & .79 \\
\hline 12 & $1-7$ & 5.82 & 1.71 & -1.53 & 1.34 & .26 & .80 \\
\hline 13 & $1-7$ & 5.92 & 1.21 & -1.22 & 1.69 & .40 & .80 \\
\hline 14 & $1-7$ & 5.28 & 1.47 & -1.03 & 0.96 & .39 & .79 \\
\hline 15 & $1-7$ & 6.28 & 1.21 & -2.35 & 6.42 & .51 & .79 \\
\hline 16 & $1-7$ & 4.55 & 2.19 & -0.33 & -1.37 & .34 & .80 \\
\hline 17 & $1-7$ & 4.95 & 1.65 & -0.76 & 0.06 & .36 & .80 \\
\hline 18 & $1-7$ & 6.01 & 1.24 & -1.85 & 4.06 & .35 & .80 \\
\hline 19 & $1-7$ & 5.72 & 1.80 & -1.46 & 1.13 & .29 & .80 \\
\hline 20 & $1-7$ & 4.79 & 1.86 & -0.48 & -0.87 & .42 & .79 \\
\hline 21 & $1-7$ & 4.91 & 1.85 & -0.62 & -0.66 & .42 & .79 \\
\hline Total & $37-147$ & 111.83 & 15.94 & -0.7 & 1.36 & $.80^{\mathrm{a}}$ & $.18^{b}$ \\
\hline
\end{tabular}

Note. QEWB Questionnaire for Eudaimonic Well-Being, M mean, SD standard deviation, Skew skewness, Kur kurtosis, ITC corrected item-total correlation.

${ }^{a}$ Cronbach's alpha of the total scale. ${ }^{b}$ Average interitem correlation of the total scale. 


\begin{tabular}{|c|c|c|c|c|c|c|c|c|c|c|c|c|c|c|c|c|c|c|c|c|c|}
\hline Item & 1 & 2 & 3 & 4 & 5 & 6 & 7 & 8 & 9 & 10 & 11 & 12 & 13 & 14 & 15 & 16 & 17 & 18 & 19 & 20 & 21 \\
\hline 1 & - & .31 & .04 & .30 & .17 & .34 & .07 & .25 & .34 & -.01 & .16 & .08 & .18 & .25 & .21 & .19 & .25 & .15 & .04 & .32 & .22 \\
\hline 2 & .31 & - & -.01 & .41 & .23 & .42 & .12 & .18 & .58 & -.11 & .38 & .11 & .21 & .21 & .23 & .19 & .24 & .11 & .04 & .21 & .51 \\
\hline 3 & .04 & -.01 & - & .11 & .11 & .06 & .18 & -.02 & .00 & -.02 & .08 & .09 & .06 & -.10 & .05 & .12 & .05 & -.04 & .26 & .18 & -.03 \\
\hline 4 & .30 & .41 & .11 & - & .42 & .31 & .09 & .42 & .34 & .03 & .24 & .14 & .51 & .34 & .24 & .12 & .25 & .20 & .13 & .18 & .26 \\
\hline 5 & .17 & .23 & .11 & .42 & - & .38 & .25 & .26 & .24 & .13 & .17 & .11 & .32 & .30 & .24 & .07 & .17 & .17 & .09 & .19 & .08 \\
\hline 6 & .34 & .42 & .06 & .31 & .38 & - & .20 & .18 & .47 & .05 & .36 & .01 & .26 & .27 & .53 & .27 & .28 & .25 & .13 & .28 & .37 \\
\hline 7 & .07 & .12 & .18 & .09 & .25 & .20 & - & .06 & .10 & -.03 & .23 & .25 & .08 & .19 & .28 & .25 & .03 & .15 & .25 & .22 & .10 \\
\hline 8 & .25 & .18 & -.02 & .42 & .26 & .18 & .06 & - & .18 & .11 & .11 & .08 & .42 & .19 & .33 & .05 & .25 & .38 & .11 & .09 & .14 \\
\hline 9 & .34 & .58 & .00 & .34 & .24 & .47 & .10 & .18 & - & .00 & .40 & .05 & .18 & .25 & .33 & .22 & .24 & .15 & .04 & .18 & .50 \\
\hline 10 & -.01 & -.11 & -.02 & .03 & .13 & .05 & -.03 & .11 & .00 & - & -.11 & .02 & .11 & .05 & .17 & -.05 & .07 & .18 & -.04 & -.12 & .13 \\
\hline 11 & .16 & .38 & .08 & .24 & .17 & .36 & .23 & .11 & .40 & -.11 & - & .16 & .11 & .17 & .29 & .21 & .03 & .03 & .28 & .25 & .30 \\
\hline 12 & .08 & .11 & .09 & .14 & .11 & .01 & .25 & .08 & .05 & .02 & .16 & - & .13 & .14 & .08 & .12 & .09 & .14 & .29 & .24 & .08 \\
\hline 13 & .18 & .21 & .06 & .51 & .32 & .26 & .08 & .42 & .18 & .11 & .11 & .13 & - & .31 & .34 & -.02 & .28 & .44 & .11 & .09 & .05 \\
\hline 14 & .25 & .21 & -.10 & .34 & .30 & .27 & .19 & .19 & .25 & .05 & .17 & .14 & .31 & - & .33 & .07 & .18 & .24 & .08 & .16 & .25 \\
\hline 15 & .21 & .23 & .05 & .24 & .24 & .53 & .28 & .33 & .33 & .17 & .29 & .08 & .34 & .33 & - & .14 & .25 & .38 & .17 & .12 & .20 \\
\hline 16 & .19 & .19 & .12 & .12 & .07 & .27 & .25 & .05 & .22 & -.05 & .21 & .12 & -.02 & .07 & .14 & - & .13 & .02 & .21 & .38 & .28 \\
\hline 17 & .25 & .24 & .05 & .25 & .17 & .28 & .03 & .25 & .24 & .07 & .03 & .09 & .28 & .18 & .25 & .13 & - & .35 & .01 & .20 & .19 \\
\hline 18 & .15 & .11 & -.04 & .20 & .17 & .25 & .15 & .38 & .15 & .18 & .03 & .14 & .44 & .24 & .38 & .02 & .35 & - & .14 & .09 & .05 \\
\hline 19 & .04 & .04 & .26 & .13 & .09 & .13 & .25 & .11 & .04 & -.04 & .28 & .29 & .11 & .08 & .17 & .21 & .01 & .14 & - & .35 & -.08 \\
\hline 20 & .32 & .21 & .18 & .18 & .19 & .28 & .22 & .09 & .18 & -.12 & .25 & .24 & .09 & .16 & .12 & .38 & .20 & .09 & .35 & - & .17 \\
\hline 21 & .22 & .51 & -.03 & .26 & .08 & .37 & .10 & .14 & .50 & .13 & .30 & .08 & .05 & .25 & .20 & .28 & .19 & .05 & -.08 & .17 & - \\
\hline
\end{tabular}


magnitude, and positive correlations with the sum of the remainder of the items. Itemlevel descriptive statistics also provide a way to explore the multivariate normality, an assumption for methods such as maximum likelihood CFA, on a univariate level (Bandalos and Finney 2010). For a scale that measures well-being it would, however, be sad if expected values lie near the middle value of the Likert scale. Instead, we would like the expected values to be towards the positive end of the Likert scale for positivephrased items, with scores normally distributed around those expected values. Table 1 provides the item-level descriptive statistics for all items. Table 2 provides the interitem correlation matrix. The reversed-phrased items' scores were reversed prior to these analyses for the purpose of comparability.

The mean scores for most items lay above the centre point of the scale, which indicated that responses were more to the positive end of the scale (all items' mean values were larger than 4.00, the centre point of the Likert scale, except for one item; furthermore, there were 13 items with mean scores above 5.00 ). This was confirmed by the mostly negative skewness statistics. Relatively small standard deviations for a number of items implied the limited variability of certain items. Skewness and kurtosis values of less than $|2.00|$ can be considered acceptable deviations from normality, but more liberal standards allow for values less than $|7.00|$ (Bandalos and Finney 2010). In this study, most skewness and kurtosis values were less than $|2.00|$ and all were less than $|7.00|$. There were items that had negative correlations with other items and small or negative item-total correlations, which pointed towards problems with the proposed unidimensionality of the scale (Blunch 2008).

\section{Internal consistency reliability and scale total descriptive statistics}

Cronbach's alpha coefficient, for which values larger than .70 are often deemed adequate (Kline 2011), and the average interitem correlation, which should fall between .15 and .50 (Clark and Watson 1995), were used to ascertain internal consistency reliability. These indices, together with the descriptive statistics for the total score of the QEWB, are reported in the last row of Table 1.

The reliability indices showed satisfactory internal consistency reliability of the scale. While scale total scores could range from 21 to 147 with a centre point of 84.00 in this scale, the obtained mean and range scores indicated that respondents' total scores lay more towards the high EWB end of the scale. Note that the mean total score minus 1.5 times the standard deviation of the total score was 87.92, which is still larger than 84.00. In order to compare the average scores in our sample with the mean scores reported by Waterman et al. (2010), we transposed our sample's average score by dividing it by 7 and multiplying it by 5 (for transposition from our 7-point to Waterman et al.'s 5-point scale), and then subtracting 21 from the answer (to compensate for the fact that our lowest response option was 1, while Waterman et al.'s Likert scale started at 0 ). The transposed average was 58.88 , which is slightly higher than Waterman et al.'s two samples' means of 56.83 and 54.63. Although Waterman et al. noted that their reported levels of EWB were in the moderate range and not concentrated in the upper part of the range, we are of the opinion that the mean for our sample is considerably larger than 42.00 , the centre point of the scale used by Waterman et al. This was confirmed by negative skewness values. The skewness and kurtosis values of the scale's total score was less than $|2.00|$, which could be seen as only slight departure from normality (Bandalos and Finney 2010). 


\section{Demographic comparisons for QEWB scores}

Analyses were conducted to compare the average QEWB scores for different gender and age groups.

\section{Gender. $^{a}$}

The average QEWB total score for males $(M=112.22, S D=14.43)$ was not statistically significantly larger than the average score for females $(M=111.56, S D=16.40), t(320)=.32$, $p=.75$. The effect size, Cohen's $d=0.04$, is small according to guidelines used for interpreting $d$. All item-level comparisons for gender also yielded small $d$-values.

Age. ${ }^{b}$

To compare the average QEWB total score for different age groups, the sample was divided into eight groups according to age, namely age $18(n=26, M=105.31, S D=20.51)$, $19(n=82, M=111.78, S D=16.25), 20(n=84, M=111.78, S D=15.18), 21 \quad(n=62$, $M=111.48, S D=15.46), 22(n=26, M=114.95, S D=12.44), 23-25(n=19, M=113.00$, $S D=13.85), 26-30(n=8, M=119.05, S D=11.45)$, and 31 and older $(n=12, M=118.57$, $S D=18.68)$. A one-way ANOVA indicated a statistically nonsignificant effect for age, $F(7,311)=1.34, p=.23$. The effect size, $\eta^{2}=.029$, is small, indicating that age accounted for minimal variance in QEWB scores. Effect sizes for pairwise comparisons between age groups (Cohen's $d$-values) were larger than 0.50 in the following instances: age group 31 and older versus age group $18(d=0.65)$ and age group $26-30$ versus age group $18(d=.67)$.

\section{Factor structure of the QEWB}

Waterman et al. (2010) confirmed the unifactorial structure of the QEWB by obtaining adequate fit indices when using parcelled indicators in CFA. Although this could have suggested that a higher order factor underlay the scale, we suspected that a more nuanced understanding of the scale's dimensionality might have arisen if the data was analysed on item level. We were also concerned that the crucial assumption for parcelling, namely unidimensional parcels, may not have held. We started our investigation of the scale's underlying factor structure for our sample by testing the proposed unidimensional structure of the QEWB using both parcelled and item-level indicators in CFA. We also examined loadings on the first unrotated component in principal component analysis. Possible multidimensional structures by means of EFAs were also explored.

In order to conduct EFA and CFA, the sample size must be sufficient. For this sample, the Kaiser-Mayer-Olkin (KMO) measure of sampling adequacy was .82, which is above .70 and can therefore be considered "good" (Hutcheson and Sofroniou 1999).

Table 3 Fit Indices for CFAs of Scores on the QEWB

\begin{tabular}{lllllll}
\hline CFA level & $\boldsymbol{X}^{\mathbf{2}}$ & $\boldsymbol{d f}$ & $\boldsymbol{p}$ & CFI & RMSEA & $\mathbf{9 0 \%} \mathbf{C l}$ \\
\hline Parcel-level & 9.5 & 5 & .09 & 0.99 & .053 & {$[.000, .103]$} \\
Item-level & 772.56 & 189 & $<.01$ & 0.62 & .098 & {$[.091, .105]$} \\
\hline
\end{tabular}

Note. QEWB Questionnaire for Eudaimonic Well-Being, CFA Confirmatory factor analysis, $X^{2}$ chi-square test statistic, $d f$ degrees of freedom, CFI comparative fit index, RMSEA root mean square error of approximation, $90 \% \mathrm{Cl} 90 \%$ confidence interval of the RMSEA. 


\section{Parcel-level CFA}

Waterman et al. (2010) examined the unifactorial structure of the QEWB by using five parcels, created by summating the responses to adjacent items 1-4, 5-8, 9-12, 13-16, and 17-21 as indicators in CFA. Standard structural equation modelling fit criteria indicated that deviation from a perfect fit to the data was small. We created parcels in our data similar to Waterman et al. (2010) and used CFA with maximum likelihood estimation to assess the fit of a unidimensional structure. We followed Bandalos and Finney (2010) in reporting criteria from each of the three broad classes of fit indices in Table 3: the CFI from the incremental indices group (values larger than .95 are considered a sign of good fit); the RMSEA with its associated 90\% confidence interval from the parsimonious indices group (values should fall below .05 for a well-fitting model or below .08 to show that the fit is acceptable); and the chi-square test statistic, its number of degrees of freedom, and the resulting $p$-value from the absolute indices group (small $p$-values show that the fit between the hypothesised model and the perfect fit of the data is not adequate). Note that the chi-square test tests the null hypothesis that the model holds exactly in the population, which is unrealistic. Furthermore, the chi-square test statistic tends to be large when the sample size is large, leading to rejection of the null hypothesis even if the model fits the data well. The chi-squared test is therefore reported, but not used for interpretation. Both the RMSEA and CFI values depicted in Table 3 suggested that the model fitted the data well.

In line with Kline's (2011) guideline that parcelling only suffices when items that are combined into parcels are unidimensional and that the use of parcelling when items that are combined are not unidimensional may lead to deceptive conclusions, we examined the internal consistency of the parcels by means of Cronbach's alpha coefficient. As explained by Clark and Watson (1995), "internal consistency is a necessary but not sufficient condition for . . . unidimensionality" (p. 315). For our sample, the five parcels' Cronbach's alpha scores were $.44, .52, .26, .39$, and .46. These values clearly indicate a lack of internal consistency for most parcels and by implication a lack of unidimensionality. This implies is that the parcel-level CFA fit indices in Table 3 are not interpretable.

\section{Item-level CFA}

Item-level CFAs using maximum likelihood estimation can be conducted on ordinal data if the data adhere to certain guidelines, as specified by Blunch (2008). In such a case parcelling would not be necessary. The pointers Blunch (2008) identified were satisfied in our study, since there were seven response options (which is more than the proposed minimum of five), skewness and kurtosis values on item level were within the limits of acceptable departure from normality (and therefore close to zero), and most items were slightly negatively skewed (which means that a possible limited skewness goes to the same side for all variables). By implication, commonly used estimation methods in CFA, like maximum likelihood, could be applied to our data. We therefore proceeded to conduct CFA with the items as indicators. The fit statistics, presented in Table 3, showed that a unifactorial understanding of the QEWB did not adequately fit the data.

\section{Principal component analysis to test for unidimensionality}

Following Blunch (2008) and Clark and Watson (1995), the unidimensionality of the scale was further tested with principal component analysis, where the first unrotated 
component was scrutinised. To confirm unidimensionality, the first unrotated component should account for a large proportion of the variance $(40 \%$ is an often recommended rule-of-thumb; Blunch 2008). Items should have salient loadings on the first unrotated component and items with loadings of less than .30 to .40 are candidates for removal from the scale (Clark and Watson 1995; Hair et al. 2010). Furthermore, communalities provide an index of the proportion of variance of an item that is accounted for by the component solution and, although no statistical guidelines exist to indicate exactly when a communality is "large" or "small" (Hair et al. 2010), we considered items with communalities of less than .20 to be potentially problematic.

The percentage variance explained by one component was $23.66 \%$, which is far below $40 \%$, suggesting that more than one factor was needed to explain sufficient variance. The loadings on the first unrotated component ranged from .09 to .70 in absolute value. Items 3 and 10 had component loadings of less than .30 in absolute value (.09 and .17 , respectively) on the first component and very small communalities (.01 and .03 , respectively), which may suggest that these items need to be reviewed if the scale is meant to be unidimensional. Items $7,12,16$, and 19 had component loadings between .30 and .40 in absolute value and communalities of less than .20 , which may also point towards a need for critical evaluation of the items. Items $3,7,12,16$, and 18 are negatively phrased, which could have caused confusion. Although Item 10 has a positive direction, it is phrased in a double negative manner, which could have led to uncertainty. Similar results were obtained when one factor was extracted with principal axis and maximum likelihood factor analysis.

\section{EFA}

Since the item-level CFA and principal component analysis suggested that a unidimensional factor structure did not fit our data well, we conducted EFAs using both principal axis and maximum likelihood factoring with oblimin rotation to explore the dimensionality of the items. Similar results were obtained for the two methods of factor extraction and we therefore present the results for the principal axis factor analysis only.

The point of inflection for the scree plot pointed towards three underlying factors. The pattern matrices of factor analyses with six, five, four, three, and two factors were explored for interpretability of the factors. We considered a factor a major factor when at least three items had loadings of .30 or larger on that factor and when there were no or few cross-loadings (Costello and Osborne 2005). An analysis of the rotated pattern matrices revealed that the three- and four-factor solutions were interpretable and consisted of only major factors. The five- and six-factor solutions contained factors that could not be considered major factors according to the criteria, while the two-factor solution was difficult to interpret and explained little variance.

The pattern matrices of the three- and four-factor solutions are presented in Table 4 . For the three-factor solution, we labelled the factors Sense of Purpose, Purposeful Personal Expressiveness, and Effortful Engagement. The items that loaded high on the Purposeful Personal Expressiveness factor divided into two factors in the four-factor solution, while the Sense of Purpose and Effortful Engagement factors remained the same. We labelled the two subfactors Engagement in Rewarding Activities and Living from Beliefs. The three factors explained $41.99 \%$ of the variance, while four factors explained $47.60 \%$ of the variance. 
Table 4 Pattern Matrices of Three and Four-Factor Principal Axis Factor Analyses with Oblimin Rotation and Factor Reliabilities

\begin{tabular}{|c|c|c|c|c|c|c|c|}
\hline \multirow[t]{2}{*}{ Item } & \multicolumn{3}{|c|}{$\begin{array}{l}\text { Three-factor } \\
\text { solution }\end{array}$} & \multicolumn{4}{|c|}{$\begin{array}{l}\text { Four-factor } \\
\text { solution }\end{array}$} \\
\hline & F1 & $\mathrm{F} 2$ & F3 & $\mathrm{F} 1$ & F2a & $\mathrm{F} 2 \mathrm{~b}$ & F3 \\
\hline 9. I can say that I have found my purpose in life. & .77 & & & .75 & & & \\
\hline 2. I believe I have discovered who I really am. & .72 & & & .68 & & & \\
\hline 21. I believe I know what I was meant to do in life. & .71 & & & .71 & & & \\
\hline $\begin{array}{l}\text { 6. I believe I know what my best potentials are and I try to develop } \\
\text { them whenever possible. }\end{array}$ & .50 & .25 & & .54 & .28 & & \\
\hline 11. As yet, I've not figured out what to do with my life. (R) & -.47 & & .26 & -.46 & & & .26 \\
\hline 1. I find I get intensely involved in many of the things I do each day. & .31 & .22 & & .31 & & & \\
\hline 18. It is important to me that I feel fulfilled by the activities that I engage in. & & .67 & & & .67 & & \\
\hline $\begin{array}{l}\text { 13. I believe it is important to know how what I'm doing fits with } \\
\text { purposes worth pursuing. }\end{array}$ & & .63 & & & .27 & -.56 & \\
\hline $\begin{array}{l}\text { 8. I feel best when I'm doing something worth investing a great deal of } \\
\text { effort in. }\end{array}$ & & .57 & & & .35 & -.34 & \\
\hline $\begin{array}{l}\text { 15. When I engage in activities that involve my best potentials, I have } \\
\text { this sense of really being alive. }\end{array}$ & & .48 & & .22 & .57 & & -.22 \\
\hline $\begin{array}{l}\text { 14. I usually know what I should do because some actions just feel right } \\
\text { to me. }\end{array}$ & & .39 & & & .25 & -.23 & \\
\hline 17. I find a lot of the things I do are personally expressive for me. & & .39 & & & .32 & & \\
\hline $\begin{array}{l}\text { 4. My life is centered around a set of core beliefs that give meaning to } \\
\text { my life. }\end{array}$ & .33 & .36 & & & & -.82 & \\
\hline $\begin{array}{l}\text { 5. It is more important that I really enjoy what I do than that other } \\
\text { people are impressed by it. }\end{array}$ & & .36 & & & & -.36 & \\
\hline $\begin{array}{l}\text { 10. If I did not find what I was doing rewarding for me, I do not think I } \\
\text { could continue doing it. }\end{array}$ & & .30 & & & .34 & & \\
\hline 19. If something is really difficult, it probably isn't worth doing. (R) & & & .67 & & & & .66 \\
\hline 20. I find it hard to get really invested in the things that I do. (R) & & & .55 & & & & .54 \\
\hline $\begin{array}{l}\text { 7. Other people usually know better what would be good for me to do } \\
\text { than I know myself. (R) }\end{array}$ & & & .47 & & & & .47 \\
\hline $\begin{array}{l}\text { 12. I can't understand why some people want to work so hard on the } \\
\text { things that they do. (R) }\end{array}$ & & & .39 & & & & .39 \\
\hline 3. I think it would be ideal if things came easily to me in my life. (R) & & & .38 & & & & .38 \\
\hline 16. I am confused about what my talents really are. (R) & -.31 & & .35 & -.34 & & & .34 \\
\hline Cronbach's alpha coefficient & .77 & .73 & .61 & .77 & .51 & .71 & .61 \\
\hline Average interitem correlation & .33 & .26 & .24 & .33 & .25 & .33 & .24 \\
\hline
\end{tabular}

Note. QEWB Questionnaire for Eudaimonic Well-Being, $(R)$ Reversed-phrased item, F1 Sense of Purpose, F2 Purposeful Personal Expressiveness, F3 Effortful Engagement, F2a Engagement in Rewarding Activities, F2 $b$ Living from Beliefs. Factor loadings $\leq .20$ are suppressed. The factor to which an item was assigned based on factor loading and interpretability is indicated in boldface. Cronbach's alpha coefficient and the average interitem correlation were calculated accordingly for each factor.

The factor to which each item was assigned for the calculation of factor scores is indicated in gray shadow in Table 4. If an item loaded high on more than one factor, we assigned the item to one of the factors based on the magnitude of the factor loadings and interpretability. Cronbach's alpha coefficient and the average interitem correlation, also presented in Table 4, were calculated for each factor as measures of internal consistency reliability. Although the Cronbach's alpha coefficients of the Effortful Engagement and Engagement in Rewarding Activities factors were below the .70 guideline, the average interitem correlations lay within the proposed $.15-.50$ range for all 
factors. All corrected item-total correlations were larger than .20, except for Item 10, which had a corrected item-total correlation of .18 in the Purposeful Personal Expressiveness factor in the three-factor solution and of .19 in the Engagement in Rewarding Activities subfactor in the four-factor solution. With the removal of Item 10, these factors' Cronbach's alpha coefficients increased to .77 and .60, respectively. Considering the fact that all average interitem correlations were above .15, we decided to retain all items, including Item 10, for the calculation of factor scores. The performance of Item 10 should be scrutinised in further studies. Factor scores were calculated as the average score of the items assigned to the factor after reversal of reversed phrased questions' scores.

After reversal of the reversed phrased questions' scores, the correlations between the Sense of Purpose factor in the three-factor solution and the Purposeful Personal Expressiveness and Effortful Engagement factors were .28 and .30, respectively, while the correlation between the Purposeful Personal Expressiveness and Effortful Engagement factors was .19. For the four-factor solution, the Sense of Purpose factor had correlations of .17, .29, and .28 with the Engagement in Rewarding Activities, Living from Beliefs, and Effortful Engagement factors, respectively. The Engagement in Rewarding Activities factor had correlations of .37 and .10 with the Living from Beliefs and Engagement in Rewarding Activities factors, respectively, The Living from Beliefs and Engagement in Rewarding Activities factors had a correlation of .23.

\section{External validity of the QEWB}

Table 5 contains the attenuation corrected Pearson correlations between scores on the QEWB factors and measures of psychological well-being and dysfunction. For the purpose of comparability with correlations reported in other studies, the noncorrected correlations are also provided in Table 5. However, we interpret and discuss only the attenuation corrected correlations in this article, since it compensates for unreliability of the measures. For the remainder of the article the term "correlation" will be used to refer to "attenuation corrected correlation". Furthermore, correlations between the

Table 5 Attenuation Corrected and Uncorrected Pearson Correlations between Scores on the QEWB Factors and Criterion Measures

\begin{tabular}{lllllll}
\hline Criterion measure & QEWB & QEWB-F1 & QEWB-F2 & QEWB-F3 & QEWB-F2a & QEWB-F2b \\
\hline MHC-SF & $.63\left(.52^{* * *}\right)$ & $.74\left(.61^{* * *}\right)$ & $.39\left(.31^{* * *}\right)$ & $.26\left(.19^{* * *}\right)$ & $.30\left(.20^{* * *}\right)$ & $.41\left(.32^{* * *}\right)$ \\
MHC-SF-EWB & $.53\left(.41^{* *}\right)$ & $.56\left(.43^{* * *}\right)$ & $.35\left(.26^{* * *}\right)$ & $.29\left(.20^{* * *}\right)$ & $.32\left(.20^{* * *}\right)$ & $.32\left(.24^{* * *}\right)$ \\
MHC-SF-PWB & $.73\left(.58^{* *}\right)$ & $.83\left(.65^{* * *}\right)$ & $.46\left(.35^{* * *}\right)$ & $.37\left(.25^{* * *}\right)$ & $.32\left(.20^{* * *}\right)$ & $.51\left(.38^{* * *}\right)$ \\
MHC-SF-SWB & $.40\left(.31^{* * *}\right)$ & $.55\left(.41^{* *}\right)$ & $.23\left(.17^{* *}\right)$ & $.07(.05)$ & $.19\left(.11^{*}\right)$ & $.24\left(.17^{* *}\right)$ \\
SWLS & $.53\left(.41^{* * *}\right)$ & $.64\left(.48^{* * *}\right)$ & $.29\left(.21^{* * *}\right)$ & $.27\left(.18^{* *}\right)$ & $.17(.11)$ & $.35\left(.25^{* * *}\right)$ \\
MLQ-P & $.80\left(.66^{* *}\right)$ & $.96\left(.77^{* * *}\right)$ & $.45\left(.35^{* * *}\right)$ & $.37\left(.27^{* * *}\right)$ & $.36\left(.24^{* * *}\right)$ & $.46\left(.36^{* * *}\right)$ \\
MLQ-S & $-.07(-.06)$ & $-.13(-.10)$ & $.06(.05)$ & $-.10(-.07)$ & $.02(.01)$ & $.09(.07)$ \\
SOC & $.62\left(.50^{* * *}\right)$ & $.64\left(.51^{* * *}\right)$ & $.35\left(.27^{* * *}\right)$ & $.46\left(.33^{* * *}\right)$ & $.28\left(.18^{* * *}\right)$ & $.35\left(.27^{* * *}\right)$ \\
PHQ-9 & $-.42\left(-.34^{* * *}\right)$ & $-.43\left(-.34^{* * *}\right)$ & $-.20\left(-.15^{* *}\right)$ & $-.37\left(-.26^{* * *}\right)$ & $-.17\left(-.11^{*}\right)$ & $-.19\left(-.14^{* * *}\right)$ \\
\hline
\end{tabular}

Notes. In each cell the attenuation corrected Pearson correlations are given first, followed by the uncorrected Pearson correlations in brackets. QEWB Questionnaire for Eudaimonic Well-Being, QEWB-F1 QEWB-Sense of Purpose, QEWB-F2 QEWBPurposeful Personal Expressiveness, QEWB-F3 QEWB-Effortful Engagement, QEWB-F2a QEWB-Engagement in Rewarding Activities, QEWB-F2b QEWB-Living from Beliefs. MHC-SF Mental Health Continuum - Short Form, MHC-SF-EWB MHC-SF Emotional Well-Being, MHC-SF-PWB MHC-SF Psychological Well-Being, MHC-SF-SWB MHC-SF Social Well-Being, SWLS Satisfaction with Life Scalem, MLQ-P Meaning in Life Questionnaire Presence, MLQ-S Meaning in Life Questionnaire Search, SOC Sense of Coherence Scale, PHQ-9 Patient Health Questionnaire-9. ${ }^{* *} p<.001 .{ }^{* *} p<.01 .{ }^{*} p<.05$. 
QEWB total score and the criterion scales' scores are presented for the sake of completeness, but, since we found that a unidimensional structure of the QEWB did not fit the data well, we do not interpret these correlations. Rather, we focus on the correlations between the attained QEWB factors' scores and scores on the criterion scales for interpretation. The QEWB factors had larger positive correlations with scores on other measures in the family of EWB (Mental Health Continuum - Short Form: Psychological Well-Being, Meaning in Life Questionnaire: Presence, and Sense of Coherence Scale) than with scores on measures more associated with hedonic well-being (Mental Health Continuum - Short Form: Emotional Well-Being and Satisfaction with Life Scale). Negative correlations were found with scores on an index of depression (Patient Health Questionnaire - 9). There were small negative correlations between the Sense of Purpose and Effortful Engagement factors' scores and the Meaning in Life Questionnaire: Search scores, which is in line with Steger et al.'s (2006) negative correlations between the scores on this subscale and measures tapping presence of meaning in life.

\section{Discussion}

The first aim of this research was to explore the structural validity of the QEWB as proposed by Waterman et al. (2010). Analyses indicated a three-factor structure consisting of the dimensions of Sense of Purpose, Purposeful Personal Expressiveness, and Effortful Engagement, or a four-factor structure where the Purposeful Personal Expressiveness factor divided into Engagement in Rewarding Activities and Living from Beliefs factors. These findings provided support for our hypothesis that a multidimensional factor structure would better explain the data.

Our second aim was to build on Waterman et al.'s (2010) foundation and further explore the scale's external validity (convergent and discriminant) with specific focus on the attenuation corrected correlations between scores on the QEWB factors' scores and scores on other well-being scales, as well as a measure of psychological dysfunction. Our hypotheses were supported: Convergent validity was confirmed by large positive correlations with scores on other measures in the family of EWB, while discriminant validity was shown by medium to large (but consistently smaller) positive correlations with scores on measures of hedonic well-being, the medium to large negative (but consistently smaller) correlations with scores on a depression scale, and the small negative correlations with search for meaning.

Regarding the first aim of the study, findings showed support for a one-dimensional factor structure at parcel level (as done by Waterman et al. 2010), but also revealed that the use of parcelling was not justified as the assumption of unidimensionality within parcels was not satisfied. A lack of fit of the unidimensional factor structure was shown by a number of small or negative interitem and item-total correlations, inadequate fit indices for item-level CFA, and the small proportion of variance explained by the first unrotated component in principal component analysis, as well as small component loadings and communalities for a number of items.

With regard to the descriptive statistics, reliability indices and principal component analyses to assess the unidimensionality of the scale, some remarks are warranted. Individual items and the scale's total score yielded average values above the centre point, which is in line with what we would hope for a scale measuring well-being. Although most individual items and the scale's total score were negatively skewed, skewness and 
kurtosis statistics pointed towards sufficient normality. The small component loadings of certain items (especially Items 3 and 10) on the first unrotated component in principal component analysis, as well as their small communalities, suggest that these items may need revision if the scale is expected to be unidimensional. Item 10's corrected item-total correlations were also small in the multifactor solutions. However, both items had salient loadings on major factors in the multifactor solutions and all factors' average interitem correlations were above .15 . We decided to retain the items in the scale, but flagged Item 10 for further critical investigation in future studies. A number of items had cross-loadings (i.e., multiple loadings larger than .30) in the multifactor solutions and, if the aim is to establish a scale with related but distinct subscales, these items may need to be reworked. Furthermore, some items had nonsalient primary loadings (i.e., loadings on all factors smaller than .30) and may need modification.

A three-factor structure emerged and the moderately correlated latent dimensions were sense of purpose, purposeful personal expressiveness, and effortful engagement. In the four-factor solution, the purposeful personal expressiveness factor divided into two separate factors. The four-factor solution explains more variance than the three-factor solution, which makes it an attractive option. On the other hand, the parsimoniousness of the three-factor solution makes the three-factor solution an elegant choice. For the sake of parsimony, we propose the three-factor solution as main model in the current study. Further research utilising data from other groups is recommended to obtain clarity in the choice between the three- and four-factor structures. Linkages were found between the dimensions obtained in this study and theory in EWB literature. The dimensions also related to the six interlinked categories of EWB defined by Waterman et al. (2010), which served as basis for item content in the QEWB. Although Waterman et al. did not specify the categories to which the items belong, we related the items to the categories according to our own judgement.

Items that loaded high on the Sense of Purpose factor were concerned with especially self-knowledge and a sense of purpose in life. The items linked particularly with Waterman et al.'s (2010) categories of self-discovery and sense of purpose and meaning in life (e.g. Items 2 and 9). An item from the intense involvement in activities category (Item 1) also featured in this factor. A sense of meaning and purpose in life is often considered an integral part of well-being in EWB literature. For example, Steger et al.'s (2006) presence of meaning in life construct, which involves the subjective sense that one lives a meaningful life, Ryff's (1989) purpose in life construct, which has to do with a comprehension of life's purpose, goal directedness, and intentionality, and Wong's (2010, 2011) meaning-centred orientation towards therapy and well-being resonate with the content of this factor.

Items that loaded high on the Purposeful Personal Expressiveness factor concerned full and active engagement in activities that the individual finds meaningful. Waterman et al.'s (2010) categories of intense involvement in activities (e.g., Item 18) and enjoyment of activities as personally expressive (e.g., Item 5) were especially recognised in items that loaded on this factor. The perceived development of one's best potentials (Items 6 and 15) and investment of significant effort in pursuit of excellence (Item 8) categories also contributed to this factor. The content of this factor resonates with Fowers' (2012a, 2012b) ideas on a virtue ethics perspective on eudaimonia and flourishing as ongoing participation in characteristically human goods, such as knowledge and belonging, through 
virtuous activities. This factor is also related to the notions of intrinsic motivation, which involves the pursuit of an activity because of its inherent interest, and autonomy, which entails that a person reflectively embraces activities as his or her own, in the selfdetermination theory (Ryan et al. 2008). This factor divided into two separate factors in the four-factor solution. The first, which we labelled Engagement in Rewarding Activities, entails the value the individual places on doing things that he or she finds fulfilling, rewarding, and personally expressive and resonates strongly with Waterman's work on personal expressiveness (cf. Waterman, 1993). The second, labelled Living from Beliefs, involves the importance of living from one's convictions and could be linked with Fowers' (2012a, 2012b) virtue ethics perspective and the notion of autonomy in the selfdetermination theory (Ryan et al. 2008).

For the Effortful Engagement factor, it is noteworthy that high factor loadings (> .30) came exclusively from reversed-phrased items. In fact, all reversed-phrased items had loadings larger than .20 on this factor. The question is whether the reversed-phrased items all loaded high on a single factor due to conceptual similarity, or whether methodological issues also played a role? Conceptually, the Effortful Engagement factor appears to represent a willingness to put effort into matters, even if it is difficult, and to take responsibility for one's life direction. Waterman et al.'s (2010) categories of investment of significant effort in pursuit of excellence (e.g., Item 19), intense involvement in activities (e.g., Item 20), and self-discovery (e.g., Item 7) are especially prominent in the items that loaded high on this factor. The content of this factor resonates with ideas on flow and optimal experience (Delle Fave and Massimini 2005; Nakamura and Csikszentmihalyi 2009), which involves the experience of complete absorption in the present moment when attention is fully invested in the task at hand, a state of deep engagement and concentration where challenges and skills meet. When considered as representative of a negative construct, this factor relates to Delle Fave and Massimini's understanding of apathy as the negative pole of experience fluctuation, characterised by disengagement and a disruption of attention. Methodologically, reversed-phrased items are often critisized for causing confusion, for being complicated and for grouping together in factor analyses, against theoretical expectations. Because of these reasons, it is not uncommon for experts to recommend the use of only positive-phrased items in measurement instruments (DeVellis 2012). It is possible that methodological, rather than theoretical reasons, contributed to the high loadings of all reversed-phrased items on one factor in this study. This effect may have been aggrevated by the fact that most of the respondents $(82 \%)$ were not home language English speakers, which may have lead to increased difficulty with complexly formulated reversed-phrased items. Future studies can explore the performance of the QEWB with omitting the negatively phrased items.

In the exploration of the QEWB's external validity, the Sense of Purpose factor had larger correlations (in absolute value) with the criterion scales' scores than any of the other QEWB factors. This may suggest that the items contained in this factor may be used instead of the full scale without losing too much information, especially if the aim is to develop a unidimensional scale of EWB. In particular, this factor was extremely highly correlated with the Presence subscale of the Meaning in Life Questionnaire, which also taps a sense of meaning and purpose in life. It also had very large correlations with the Psychological Well-Being subscale of the Mental Health Continuum - Short Form and 
the Sense of Coherence Scale, which can both be considered to measure elements of EWB. This may indicate that Sense of Purpose is a very important indicator of general EWB. The factor's large positive correlations with scores on the Emotional Well-Being and Social Well-Being subscales of the Mental Health Continuum - Short Form and the Satisfaction with Life Scale, as well as its medium to large negative correlation with scores on the Patient Health Questionnaire - 9, may indicate that a high sense of purpose can also be associated with general wellness, including high hedonic well-being, and may perhaps even be a protective factor against the development of psychological illness. However, the data at hand are correlational and such causal relationships will have to be confirmed in experimental studies. The higher correlations between scores on the Sense of Purpose factor and the criterion scales could, however, also have been a product of the specific selection of criterion scales that were included in this study. Based on the preliminary findings about the QEWB's multidimensionality from this study, a first step towards developing a better understanding of how the different dimensions of EWB measured by the QEWB fits together, would be to include a broader variety of EWB scales in the research battery. In particular, scales from the EWB literature that are theoretically related to the various dimensions of the QEWB, as mentioned in the discussion above, need to be included.

\section{Limitations and directions for future research}

This study had some limitations. Firstly, the sample in this study had restrictions. The entire sample consisted of university students, who were, although ethnically diverse, homogeneous in terms of educational background. Although the sample was comparable to Waterman et al.'s (2010) exclusively student samples, future research should validate the scale for a broader population. The sample was a convenience sample, making inference to other groups impossible. However, the study still provides valuable preliminary information about the validity of the scale. Secondly, the QEWB and all criterion measures were self-report measures and behavioural measures of the constructs will be valuable. Thirdly, this study did not include specifically a cross-cultural evaluation of the applicability of Waterman et al.'s conceptualisation of EWB within the South African context. Investigation of the cross-cultural applicability of the scale in the diverse cultural groups in South Africa will be of much value.

\section{Conclusions}

In summary, explicating the nature and concomitants of EWB is currently at the forefront of research on a fulfilling life and functioning well. However, as indicated by Waterman et al. (2010), the strength of research conducted on constructs is dependent on the quality of instrumentation. The current study contributed to a further validation of the QEWB, and highlighted its multidimensional structure. Further evaluation of the scale on theoretical and empirical levels will guide the adaptation of the current scale, also specifically for applicability within the South African multicultural context. We recommend a meticulous investigation of the scale on item level, focusing on univariate psychometric properties and on the performance of the items via item response theory. Findings with regard to the multidimensionality of the scale have to be cross-validated in other studies and scrutinised on both theoretical and empirical levels with consideration of the socio-cultural context. 


\section{Endnotes}

${ }^{a}$ The sample in this study consisted of $74 \%$ females, which is similar to the two validation samples used by Waterman et al. (2010) that consisted of $76 \%$ and $74 \%$ females, respectively. Since all of the item-level comparisons for gender in this study yielded small effect sizes, the authors are not too concerned about the uneven gender distribution. However, future research should be used to confirm the findings for males and females separately. In this study, the male subsample is too small to conduct such split analyses.

b In terms of eudaimonic identity theory (Waterman 1992, 2004, 2007), which formed the theoretical base of this scale, eudaimonic well-being and its relationships with other variables may be influenced by developmental factors. Although the effect size for the influence of age on the average QEWB score was small in this sample, we detected a tendency for scores on the QEWB to be higher for older people. We therefore repeated all analyses that will be reported on in the rest of this paper for the group containing only respondents of typical student age (age $18-22, n=280$ ). All findings for this group were similar to the findings for the entire sample.

\footnotetext{
Abbreviations

QEWB: Questionnaire for Eudaimonic Well-Being; EWB: Eudaimonic well-being; CFA: Confirmatory factor analysis; EFA: Exploratory factor analysis; CFI: Comparative fit index; RMSEA: Root mean square error of approximation; $\mathrm{Cl}$ : Confidence interval.
}

\section{Competing interests}

The authors declare that they have no competing interests.

\section{Author's contributions}

LS participated in the design and planning of the study and data gathering. She performed the statistical analyses and drafted the manuscript. MPW coordinated the study, provided funding for the expenses, participated in the design and planning of the study, and provided continuous feedback regarding the draft document. IPK participated in the design and planning of the study, participated in data gathering, and provided critical feedback on the manuscript. All authors read and approved the final manuscript.

\section{Acknowledgements}

This study was financially supported by the National Research Foundation. The opinions expressed and conclusions arrived at are those of the authors and are not necessarily to be attributed to the agencies funding this study. We thank Suria Ellis for advice regarding the statistical analyses and two anonymous reviewers for their comments.

Received: 26 June 2013 Accepted: 23 September 2013

Published: 01 Oct 2013

\section{References}

Adewuya, AO, Ola, BA, \& Afolabi, OO. (2006). Validity of the Patient Health Questionnaire (PHQ-9) as a screening tool for depression amongst Nigerian university students. Journal of Affective Disorders, 96, 89-93. doi:10.1016/j. jad.2006.05.021.

Antonovsky, A. (1987). Unraveling the mystery of health: How people manage stress and stay well. San Francisco, CA: Jossey-Bass.

Antonovsky, A. (1993). The structure and properties of the Sense of Coherence Scale. Social Science \& Medicine, 36(6), 725-733. doi:10.1016/0277-9536(93)90033-Z.

Bandalos, DL, \& Finney, SJ. (2010). Factor analysis: Exploratory and confirmatory. In GR Hancock \& RO Mueller (Eds.), The reviewer's guide to quantitative methods in the social sciences (pp. 93-114). New York, NY: Routledge Taylor \& Francis.

Blunch, NJ. (2008). Introduction to structural equation modelling using SPSS and AMOS. Thousand Oaks, CA: Sage.

Botha, MN. (2011). Validation of the Patient Health Questionnaire (PHQ-9) in an African context (Master's thesis). Potchefstroom: North-West University.

Bryant, FB, King, SP, \& Smart, CM. (2007). Multivariate statistical strategies for construct validation in positive psychology. In AD Ong \& MHM van Dulmen (Eds.), Oxford handbook of methods in positive psychology (pp. 61-82). New York, NY: Oxford University Press.

Clark, LA, \& Watson, D. (1995). Constructing validity: Basic issues in objective scale development. Psychological Assessment, 7(3), 309-319. doi:10.1037/1040-3590.7.3.309.

Costello, AB, \& Osborne, JW. (2005). Best practices in exploratory factor analysis: Four recommendations for getting most from your analysis. Practical Assessment, Research \& Evaluation, 10(7), 1-9.

De Lima Osório, F, Mendes, AV, Crippa, JA, \& Loureiro, SR. (2009). Study of the discriminative validity of the PHQ-9 and PHQ-2 in a sample of Brazilian women in the context of primary health care. Perspectives in Psychiatric Care, 
45(3), 216-227. doi:10.1111/j.1744-6163.2009.00224.x.

Deci, EL, \& Ryan, RM. (2008). Hedonia, eudaimonia, and well-being: An introduction. Journal of Happiness Studies, 9(1), 1-11. doi:10.1007/s10902-006-9018-1.

Delle Fave, A, Brdar, I, Freire, T, Vella-Brodrick, D, \& Wissing, MP. (2011). The eudaimonic and hedonic components of happiness. Qualitative and quantitative findings Social Indicators Research, 100(2), 185-207. doi:10.1007/s11205-010-9632-5.

Delle Fave, A, \& Massimini, F. (2005). The investigation of optimal experience and apathy: Developmental and psychosocial implications. European Psychologist, 10(4), 264-274. doi:10.1027/1016-9040.10.4.264.

DeVellis, RF. (2012). Scale development: Theory and applications (3rd ed.). Thousand Oaks, CA: Sage.

Diener, E. (1984). Subjective well-being. Psychological Bulletin, 95(3), 542-575.

Diener, E, Emmons, RA, Larsen, RJ, \& Griffin, S. (1985). The Satisfaction With Life Scale. Journal of Personality Assessment, 49(1), 71.

Fowers, BJ. (2012a). An Aristotelian framework for the human good. Journal of Theoretical and Philosophical Psychology, 32(1), 10-23. doi:10.1037/a0025820

Fowers, BJ. (2012b). Placing virtue and the human good in psychology. Journal of Theoretical and Philosophical Psychology, 32(1), 1-9. doi:10.1037/a0025819.

Hair, JF, Black, WC, Babin, BJ, Anderson, RE, \& Tatham, RL. (2010). Multivariate data analysis (7th ed.). Upper Saddle River, NJ: Pearson Prentice Hall.

Huta, V, \& Ryan, R. (2010). Pursuing pleasure or virtue: The differential and overlapping well-being benefits of hedonic and eudaimonic motives. Journal of Happiness Studies, 11, 735-762. doi:10.1007/s10902-009-9171-4.

Hutcheson, GD, \& Sofroniou, N. (1999). The multivariate social scientist. London, England: Sage.

Kahneman, D, Diener, E, \& Schwarz, N. (1999). Well-being: The foundations of hedonic psychology. New York, NY: Russell Sage.

Kashdan, TB, Biswas-Diener, R, \& King, LA. (2008). Reconsidering happiness: The costs of distinguishing between hedonics and eudaimonia. Journal of Positive Psychology, 3, 219-233.

Keyes, CLM. (2009). Brief description of the mental health continuum short form (MHC-SF). http://calmhsa.org/wp-content/ uploads/2013/06/MHC-SFEnglish.pdf. Accessed 17 June 2013.

Keyes, CLM. (1998). Social well-being. Social Psychology Quarterly, 61(2), 121-140.

Keyes, CLM. (2002). The mental health continuum: From languishing to flourishing in life. Journal of Health \& Social Behavior, 43(2), 207-222.

Keyes, CLM. (2006). If health matters: When will we stop saying one thing and doing another? Keynote address presented at the South African Conference on Positive Psychology: Individual, social and work wellness. South Africa: Potchefstroom

Keyes, CLM, Wissing, MP, Potgieter, JP, Temane, QM, Kruger, A, \& Van Rooy, S. (2008). Evaluation of the Mental Health Continuum-Short Form (MHC-SF) in Setswana-speaking South Africans. Clinical Psychology \& Psychotherapy, 15(3), 181-192. doi:10.1002/cpp.572.

Kline, RB. (2005). Principles and practice of structural equation modeling (2nd ed.). New York, NY: Guilford Press.

Kline, RB. (2011). Principles and practice of structural equation modeling (3rd ed.). New York, NY: Guilford Press.

Kroenke, K, Spitzer, RL, \& Williams, JBW. (2001). The PHQ-9. Journal of General Internal Medicine, 16(9), 606-613. doi:10.1046/j.1525-1497.2001.016009606.x.

Lamers, SMA, Westerhof, GJ, Bohlmeijer, ET, ten Klooster, PM, \& Keyes, CLM. (2011). Evaluating the psychometric properties of the Mental Health Continuum-Short Form (MHC-SF). Journal of Clinical Psychology, 67(1), 99-110. doi:10.1002/jclp.20741.

Liu, S, \& Gan, Y. (2010). Reliability and validity of the Chinese version of the Meaning in Life Questionnaire [Abstract]. Chinese Mental Health Journal, 24(6), 478-482.

Nakamura, J, \& Csikszentmihalyi, M. (2009). Flow theory and research. In CR Snyder (Ed.), SJ Lopez (pp. 195-206). New York, NY: Oxford University Press.

Pavot, W, \& Diener, E. (1993). Review of the Satisfaction With Life Scale. Psychological Assessment, 5(2), 164-172. doi:10.1037/1040-3590.5.2.164.

Pavot, W, \& Diener, E. (2008). The Satisfaction With Life Scale and the emerging construct of life satisfaction. The Journal of Positive Psychology, 3(2), 137-152. doi:10.1080/17439760701756946.

Ryan, RM, Huta, V, \& Deci, EL. (2008). Living well: A self-determination theory perspective on eudaimonia. Journal of Happiness Studies, 9(1), 139-170. doi:10.1007/s10902-006-9023-4.

Ryff, CD. (1989). Happiness is everything, or is it? Explorations on the meaning of psychological well-being. Journal of Personality and Social Psychology, 57(6), 1069-1081. doi:10.1037/0022-3514.57.6.1069.

Selin, H, \& Davey, G. (2012). Introduction. In H Selin \& G Davey (Eds.), Happiness across cultures: Views of happiness and quality of life in non-Western cultures (pp. 1-12). New York, NY: Springer. doi:10.1007/978-94-007-2700-7_1.

Simms, LJ. (2008). Classical and modern methods of psychological scale construction. Social and Personality Psychology Compass, 2(1), 414-433. doi:10.1111/j.1751-9004.2007.00044.x

Simões, A, Oliveira, AL, Lima, MP, Vieira, CMC, \& Nogueira, SM. (2010). Instrument to evaluate the meaning of life [Abstract]. Psicologia Educação Cultura, 14(2), 247-268.

Steger, MF, Frazier, P, Oishi, S, \& Kaler, M. (2006). The Meaning in Life Questionnaire: Assessing the presence of and search for meaning in life. Journal of Counseling Psychology, 53(1), 80-93. doi:10.1037/0022-0167.53.1.80.

Strümpfer, DJW, \& Wissing, MP. (1998). Review of South African data on the sense of coherence scale as a measure of fortigenesis and salutogenesis. Cape Town, South Africa: Paper presented at the 4th Annual Congress of the Psychological Society of South Africa.

Wang, M, \& Dai, X. (2008). Chinese Meaning in Life Questionnaire Revised in college students and its reliability and validity test [Abstract]. Chinese Journal of Clinical Psychology, 16(5), 459-461.

Waterman, AS. (1992). Identity as an aspect of optimal psychological functioning. In GR Adams, T Gullota, \& R Motemayor (Eds.), Identity formation during adolescence: Advances in adolescent development (Vol. 4, pp. 50-72). Newbury Park, CA: Sage.

Waterman, AS. (1993). Two conceptions of happiness: Contrasts of personal expressiveness (eudaimonia) and hedonic enjoyment. Journal of Personality and Social Psychology, 64(4), 678-691. doi:10.1037/0022-3514.64.4.678. 
Waterman, AS. (2004). Finding someone to be: Studies on the role of intrinsic motivation in identity formation. Identity: An International Journal of Theory and Research, 4, 209-228.

Waterman, AS. (2007). Doing well: The relationship of identity status to three conceptions of well-being. Identity: An International Journal of Theory and Research, 7, 289-308.

Waterman, AS, Schwartz, SJ, Zamboanga, BL, Ravert, RD, Williams, MK, Agocha, MB, \& Donnellan, VB. (2010). The Questionnaire for Eudaimonic Well-Being: Psychometric properties, demographic comparisons, and evidence of validity. The Journal of Positive Psychology, 5(1), 41-61. doi:10.1080/17439760903435208.

Weijters, B, Cabooter, E, \& Schillewaert, N. (2010). The effect of rating scale format on response styles: The number of response categories and response category labels. International Journal of Research in Marketing, 27(3), 236-247. doi:10.1016/j.jiresmar.2010.02.004.

Wissing, MP, Thekiso, SM, Stapelberg, R, Van Quickelberge, L, Choabi, P, Moroeng, C, \& Vorster, HH. (2010). Validation of three Setswana measures for psychological wellbeing. SA Journal of Industrial Psychology, 36(2), 1-8. doi:10.4102/ sajip.v36i2.860.

Wissing, MP, \& Van Eeden, C. (2002). Empirical clarification of the nature of psychological well-being. South African Journal of Psychology, 32(1), 32-44.

Wong, PTP. (2010). Meaning therapy: An integrative and positive existential psychotherapy. Journal of Contemporary Psychotherapy, 40(2), 85-93. doi:10.1007/s10879-009-9132-6.

Wong, PTP. (2011). Positive psychology 2.0: Towards a balanced interactive model of the good life. Canadian Psychology, 52(2), 69-81. doi:10.1037/a0022511.

10.1186/2211-1522-3-3

Cite this article as: Schutte et al:: Further validation of the questionnaire for eudaimonic well-being (QEWB).

Psychology of Well-Being: Theory, Research and Practice 2013, 3:3

Submit your manuscript to a SpringerOpen ${ }^{\circ}$ journal and benefit from:

- Convenient online submission

- Rigorous peer review

- Immediate publication on acceptance

- Open access: articles freely available online

- High visibility within the field

- Retaining the copyright to your article

Submit your next manuscript at $\boldsymbol{~ s p r i n g e r o p e n . c o m ~}$ 